\title{
A rare case of congenital fibre type disproportion causing delayed motor milestones
}

\author{
R Waduge ${ }^{1}$, N V I Ratnatunga ${ }^{1}$, A Padeniya ${ }^{2}$, D N K Bowala ${ }^{1}$, S Chandraprabha ${ }^{1}$ \\ Ceylon Medical Journal 2015; 60: 33-34
}

\section{Introduction}

Congenital myopathies are a clinically, genetically and pathologically heterogeneous group of muscle disorders. Congenital fiber type disproportion (CFTD) is a rare type of congenital myopathy which presents with hypotonia, delayed motor milestones and dysmorphic facies $[1,2]$.

\section{Case report}

A two year and four month old boy who was the second child of a non-consanguineous marriage was investigated for delayed motor mile stones and dysarthria. The patient had an uneventful antenatal history. He started walking at the age of two years. There was no progressive proximal muscle weakness. Examination showed long facies and a squint. Muscle tone in both upper and lower limbs was mildly reduced. Creatine phosphokinase levels were normal, 65U/L (24-195U/L). The nerve conduction study excluded spinal muscular atrophy.

A muscle biopsy of vastus lateralis was done.

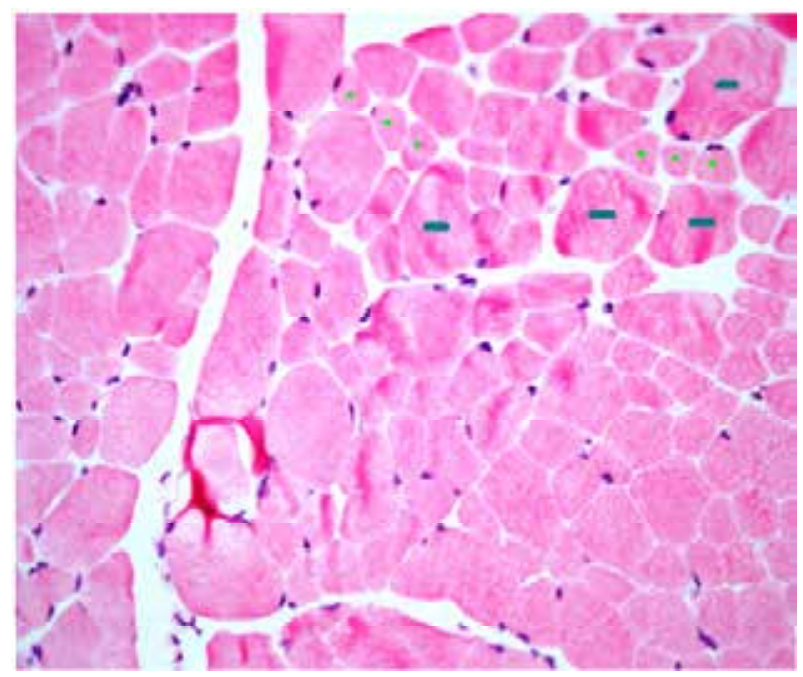

Figure 1. H \& E stain to visualise small fibres with and normal fibers with (x400)
Enzyme histochemistry for succinic dehydrogenase showed a contrasting size difference in type 1 and 2 fibres. The mean fibre diameter of type 1 and 2 fibres was $6 \mu$ and $10 \mu$ respectively. The age and sex matched norm of mean fibre diameter is $10-18 \mu$ for both type 1 and 2 fibres. The type 1 fibres occupied $64 \%$ of fibre population and type 2 fibres $36 \%$ approximately (Figures 1 and 2). No fibre necrosis, interstitial fibrosis or any other histological abnormalities were detected. Therefore a diagnosis of CFTD was made.

\section{Discussion}

The pathological criterion for determining CFTD is the predominance of type 1 fibres that are at least $12 \%$ smaller in diameter than type 2 fibres in the absence of any other pathological features [1]. In this case, the difference of mean fibre diameter of type 1 and type 2 fibres was $40 \%$. The ratio of type 1 and 2 fibres was $2: 1$ (normal ratio is 1:2) with a predominance of type 1fibres. The degree of weakness varies and some show involvement of respiratory muscles. The disease can become static or improvement can occur [1].

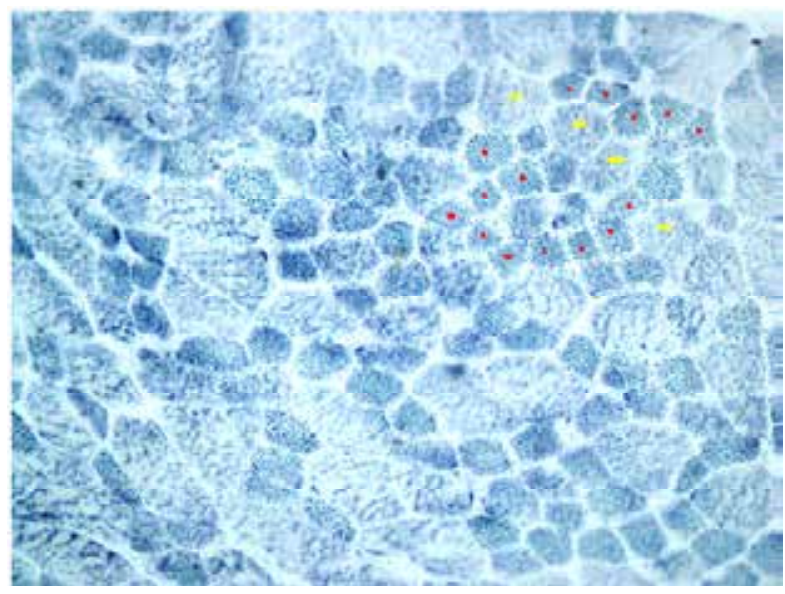

Figure 2. Succinic dehydrogenase enzyme stain to visualise darkly stained small type 1 fibres with $\square$ and lightly stained type 2 fibres with $(x 400)$

${ }^{1}$ Department of Pathology, Faculty of Medicine, University of Peradeniya and ${ }^{2}$ Sirimavo Bandaranaike Specialized Childrens Hospital, Peradeniya, Sri Lanka.

Correspondence: RW, e-mail: <roshitha2006@yahoo.com>. Received 29 August and revised version accepted 29 December 2014. Competing interests: none declared. 


\section{Declaration of Interest}

There are no conflicts of interest.

\section{References}

1. Victor Dubowitz, Caroline A Sewry, Anders Oldfors. Muscle biopsy: A practical approach, 3rd ed. Saunders 2013; 439-40

2. Mariëlle GT Bartholomeusa, Fons JM Gabreëlsa, Henk J. ter Laakb, Baziel GM van Engelenb. Congenital fibre type disproportion a time-locked diagnosis: A clinical and morphological follow-up study. Clin Neurol Neurosurg 2000; 102: 97-101. 